\title{
LEFT-INVARIANT ALMOST PARA-COMPLEX EINSTEINIAN STRUCTURES ON SIX-DIMENSIONAL NILPOTENT LIE GROUPS
}

\author{
Nikolay K. Smolentsev
}

Kemerovo State University, Krasnaya Str. 6, Kemerovo, 650043, Russian Federation

Received October 24, 2017;

e-mail: smolennk@mail.ru

Accepted in revised form November 26, 2017;

Published December 30, 2017

\begin{abstract}
As is well known, there are 34 classes of isomorphic simply connected six-dimensional nilpotent Lie groups. Of these, only 26 classes admit left-invariant symplectic structures and only 18 admit left-invariant complex structures. There are five six-dimensional nilpotent Lie groups $G$, which do not admit neither symplectic, nor complex structures and, therefore, can be neither almost pseudoKählerian, nor almost Hermitian. In this work, these Lie groups are being studied. The aim of the paper is to define new left-invariant geometric structures on the Lie groups under consideration that compensate, in some sense, the absence of symplectic and complex structures. Weakening the closedness requirement of left-invariant 2-forms $\omega$ on the Lie groups, non-degenerated 2-forms $\omega$ are obtained, whose exterior differential $d \omega$ is also non-degenerated in Hitchin sense [6]. Therefore, the Hitchin's operator $K_{d \omega}$ is defined for the 3 -form $d \omega$. It is shown that $K_{d \omega}$ defines an almost complex or almost para-complex structure for $G$ and the couple $(\omega, d \omega)$ defines pseudo-Riemannian metrics of signature $(2,4)$ or $(3,3)$, which is Einsteinian for 4 out of 5 considered Lie groups. It gives new examples of multiparametric families of Einstein metrics of signature $(3,3)$ and almost para-complex structures on six-dimensional nilmanifolds, whose structural group is being reduced to $S L(3, \mathrm{R}) \subset S O(3,3)$. On each of the Lie groups under consideration, compatible pairs of left-invariant forms $(\omega, \Omega)$, where $\Omega=d \omega$, are obtained. For them the defining properties of half-flat structures are naturally fulfilled: $d \Omega=0$ and $\omega \wedge \Omega=0$. Therefore, the obtained structures are not only almost Einsteinian para-complex, but also pseudoRiemannian half-flat.
\end{abstract}

Keywords: Nilmanifolds, six-dimensional nilpotent Lie algebras left-invariant para-complex structures, Einstein manifolds, half-flat structures

DOI $10.21603 / 2500-1418-2017-2-2-88-95$

\section{INTRODUCTION}

Left-invariant Kählerian structure on Lie group $G$ is a triple $(g, \omega, J)$ consisting of a left-invariant Riemannian metric $g$, left-invariant symplectic form $\omega$ and orthogonal left-invariat complex structure $J$, where $g(X, Y)=\omega(X, J Y)$ for any left-invariant vectors fields $X$ and $Y$ on $G$. Therefore, such a structure on $G$ can be given by a pair $(\omega, J)$, where $\omega$ is a symplectic form, and $J$ is a complex structure compatible with $\omega$, that is, such that $\omega(J X, J Y)=\omega(X, Y)$. If $\omega(X, J X)>0, \forall X \neq 0$, it is Kählerian metrics. If the positivity condition is not satisfied, then $g(X, Y)=\omega(X, J Y)$ is a pseudoRiemannian metric and then $(g, \omega, J)$ is called a pseudoKähler structure on the Lie group $G$. Classification of real six-dimensional nilpotent Lie algebras admitting invariant complex structures were obtained in [8]. Classification of symplectic structures on sixdimensional nilpotent Lie algebras was obtained in [5]. Out of 34 classes of isomorphic simply connected sixdimensional nilpotent Lie groups, only 26 admit leftinvariant symplectic structures. Condition of existence of left-invariant positively definite metric on Lie group $G$ applies restrictions to the structure of its Lie algebra g. For example, it was shown in [2] that such a Lie algebra can not be nilpotent except for the abelian case. Although nilpotent Lie groups and nilmanifolds (except for torus) do not admit Kählerian left-invariant metrics, on such manifolds left-invariant pseudoRiemannian Kählerian metrics may exist. It was shown in [4] that 14 classes of symplectic six-dimensional nilpotent Lie groups admit compatible complex structures and, therefore, define pseudo-Kähler metrics. A more complete study of the properties of the curvature of such pseudo-Kähler and almost pseudoKähler structures was carried out in $[9,10]$.

As mentioned before, 26 out of 34 classes of sixdimensional nilpotent Lie groups admit left-invariant symplectic structures. Out of last 8 classes of nonsymplectic Lie groups, 5 Lie groups $G_{i}$ do not also admit complex structures [8]; their Lie algebras $\mathbf{g}_{i}$ are shown below:

$\mathbf{g}_{1}:(0,0,12,13,14+23,34-25)$,

$\mathbf{g}_{2}:(0,0,12,13,14,34-25)$,

$\mathbf{g}_{3}:(0,0,0,12,13,14+35)$,

$\mathbf{g}_{4}:(0,0,0,12,23,14+35)$,

$\mathbf{g}_{5}:(0,0,0,0,12,15+34)$.

In this paper we study precisely these Lie groups. The aim of the paper is to define new left-invariant geometric structures on the Lie groups under consideration that compensate, in some sense, the failure of symplectic and complex structures. It is shown that on all such Lie groups $G_{i}$ any left-invariant closed 2 -form $\omega$ is degenerated. There are natural ways to weaken the closedness requirement of $\omega$ to preserve non-degeneracy $\omega$, in ways that 3 -form $d \omega$ is also non-

Please cite this article in press as: Smolentsev N.K. Left-invariant almost para-complex einsteinian structures on six-dimensional nilpotent Lie groups. Science Evolution, 2017, vol. 2, no. 2, pp. 88-95. DOI: 10.21603/2500-1418-2017-2-2-88-95.

Copyright (C) 2017, Smolentsev et al. This is an open access article distributed under the terms of the Creative Commons Attribution 4.0 International License (http:// creativecommons.org/licenses/by/4.0/), allowing third parties to copy and redistribute the material in any medium or format and to remix, transform, and build upon the material for any purpose, even commercially, provided the original work is properly cited and states its license. This article is published with open access at http:// scienceevolution.ru/. 
degenerated and property $\omega \wedge d \omega=0$ is satisfied. Hitchin's operator $K_{d \omega}$ corresponding to nondegenerated 3-form $d \omega$, can define either almost complex structure, or almost para-complex, depending on the chosen $\omega$. Associated metric $g(X, Y)=\omega\left(X, J_{d \omega} Y\right)$ is pseudo-Riemannian of signatures $(3,3)$ or $(2,4)$. The structural group is reduced to $S L(3, \mathbf{R})$ in case of signature $(3,3)$ and to $S U(1,2)$ in case of signature $(2,4)$. On groups $G_{2}-G_{5}$ this metric is Einsteinian of signature $(3,3)$. An explicit form of these metrics is presented. As a result, we obtain a compatible couple $(\omega, \Omega)$, where $\Omega=d \omega$. We present an explicit form of pseudo almost Hermitian half-flat and para-complex half-flat structures.

For any nilpotent Lie group $G$ with rational structure constants there exists a discrete subgroup $\Gamma$ such that $M=\Gamma \backslash G$ is a compact manifold called a nilmanifold. Therefore, all the results hold for the corresponding sixdimensional compact nilmanifolds.

All calculations were made in the Maple system according to the usual formulas (see eg [10]) for the geometry of left-invariant structures.

\section{MATERIALS AND METHODS}

Let $G$ be a real Lie group of dimension $m$ and $\mathbf{g}$ be its Lie algebra. Lower central series of Lie algebra $g$ is decreasing sequence of ideals $C^{0} \mathbf{g}, C^{1} \mathbf{g}, \ldots$, being defined inductively: $C^{0} \mathbf{g}=\mathbf{g}, C^{k+1} \mathbf{g}=\left[\mathbf{g}, C^{k} \mathbf{g}\right]$. Lie algebra $\mathbf{g}$ is called nilpotent, if $C^{k} \mathbf{g}=0$ for some $k$. In this case, the minimum length of lower central series is called class (or step) of nilpotency. In other words, the Lie algebra class is equal to $s$, if $C^{s} \mathbf{g}=0$ and $C^{s-1} \mathbf{g} \neq 0$. In this case, $C^{-1} \mathbf{g}$ lies in the center $Z(\mathbf{g})$ of the Lie algebra $\mathbf{g}$. The increasing central sequence $\left\{\mathbf{g}_{l}\right\}$ was defined for nilpotent $s$-step Lie algebra,

$$
\mathbf{g}_{0}=\{0\} \subset \mathbf{g}_{1} \subset \mathbf{g}_{2} \subset \cdots \subset \mathbf{g}_{s-1} \subset \mathbf{g}_{s}=\mathbf{g},
$$

where the ideals $\mathbf{g}_{l}$ were defined inductively by the rule:

$$
\mathbf{g}_{l}=\left\{X \in \mathbf{g} \mid[X, \mathbf{g}] \subseteq \mathbf{g}_{l-1}\right\}, l \geq 1 .
$$

Particularly, $\mathbf{g}_{1}$ is a center of Lie algebra. One can see from this sequence that nilpotency property is equivalent to existence of basis $\left\{e_{1}, \ldots, e_{m}\right\}$ of the Lie algebra g, for which

$$
\left[e_{i}, e_{j}\right]=\sum_{k>i, j} C_{i j}^{k} e_{k}, \quad 1 \leq i<j \leq m .
$$

Nilpotency is also equivalent to the existence of basis $\left\{e^{1}, \ldots, e^{m}\right\}$ of left-invariant 1 -forms on $G$ such that

$$
d e^{i} \in \Lambda^{2}\left\{e^{1}, \ldots, e^{i-1}\right\}, \quad 1 \leq i \leq m,
$$

where the right side is considered to be zero for $i=1$. As is known, the exterior differential of a left-invariant 1 -form is expressed through the structural constants of a Lie algebra by the formula [7]:

$$
d e^{k}=-\sum_{i<j} C_{i j}^{k} e^{i} \wedge e^{j},
$$

where $\left\{e^{1}, \ldots, e^{m}\right\}$ is the dual to $\left\{e_{1}, \ldots, e_{m}\right\}$ basis in $\mathbf{g}^{*}$. Therefore, the structure of a Lie algebra is given either by specifying nonzero Lie brackets or by differentials of basis left-invariant 1-forms. The Lie algebra $\mathbf{g}$ is often defined as an $m$-tuple based on a sequence of differentials $\left(0,0, d e^{3}, \ldots, d e^{m}\right)$ of basic 1 -forms, in the notation $i j$ is used instead of $e^{i j}=e^{i} \wedge e^{j}$. For example, notation $(0,0,0,0,12,34)$ denotes Lie algebra with structural equations: $d e^{1}=d e^{2}=d e^{3}=0, d e^{4}=0$, $d e^{5}=e^{1} \wedge e^{2}$ and $d e^{6}=e^{3} \wedge e^{4}$.

Left-invariant symplectic structure on Lie group $G$ is a left-invariant closed 2 -form $\omega$ of the maximal rank. It is given by 2-form $\omega$ of the maximal rank on Lie algebra $\mathbf{g}$. Closedness of the 2 -form is equivalent to condition

$$
\omega([X, Y], Z)-\omega([X, Z], Y)+\omega([Y, Z], X)=0, \forall X, Y, Z \in \mathbf{g} .
$$

In this case, Lie algebra $\mathbf{g}$ and group $G$ will be called symplectic ones.

Left-invariant almost complex structure on Lie group $G$ is left-invariant field of endomorphisms $J: T G \rightarrow T G$ of tangent bundle $T G$, having the property $J^{2}=-I d$. Since $J$ is defined by linear operator $J$ on Lie algebra $\mathbf{g}=T_{e} G$, we will say that $J$ is a left-invariant almost complex structure on Lie algebra g. In order for the almost complex structure $J$ to define a complex structure on the Lie group $G$, it is necessary and sufficient (according to the NewlenderNirenberg theorem [7]) that the Nijenhuis tensor vanishes:

$$
\begin{gathered}
{[J X, J Y]-[X, Y]-J[J X, Y]-J[X, J Y]=0,} \\
\text { for any } X, Y \in \mathbf{g} .
\end{gathered}
$$

For the left-invariant complex structure on Lie group $G$ left shifts $L_{g}: G \rightarrow G, g \in G$ are holomorphic.

Left-invariant Kählerian structure on Lie group $G$ is a triple $(g, \omega, J)$ consisting of a left-invariant Riemannian metric $g$, left-invariant symplectic form $\omega$ and orthogonal left-invariant complex structure $J$, where $g(X, Y)=\omega(X, J Y), \forall X, Y \in \mathbf{g}$. Therefore, such a structure on Lie group $G$ can be specified by a couple $(\omega, J)$, where $\omega$ is a symplectic form, and $J$ is a complex structure being compatible with $\omega$, i.e. such that $\omega(J X$, $J Y)=\omega(X, Y), \forall X, Y \in \mathbf{g}$. If $\omega(X, J Y)>0, \forall X \neq 0$, then it is Kählerian metric $g(X, Y)=\omega(X, J Y)$. But if the positivity condition is not fulfilled, then $g(X, Y)$ is pseudo-Riemannian metric and then $(g, J, \omega)$ is called pseudo-Kählerian structure on Lie group $G$. In further, (pseudo)Kählerian structure will be specified by pair $(J, \omega)$ of compatible left-invariant complex and symplectic structures. It follows from left-invariance that (pseudo)Kählerian structure $(g, J, \omega)$ can be given by the values $J, \omega$ and $g$ on Lie algebra $\mathbf{g}$ of the Lie group $G$. In this case $(\mathbf{g}, J, \omega, g)$ is called pseudo-Kählerian Lie algebra.

Almost para-complex structure on $2 n$-dimensional manifold $M$ is a field $P$ of endomorphisms of the tangent bundle $T M$ such that $P^{2}=I d$, where ranks of eigendistributions $T^{ \pm} M:=\operatorname{ker}(I d \pm P)$ are equal. Almost paracomplex structure $P$ is called integrable if distributions $T^{ \pm} M$ are involutive. In this case, $P$ is called paracomplex structure. A manifold $M$ supplied by (almost) para-complex structure $P$, is called (almost) paracomplex manifold. The Nijenhuis tensor $N_{P}$ of almost para-complex structure $P$ is defined by equation

$$
N_{P}(X, Y)=[X, Y]+[P X, P Y]-P[P X, Y]-P[X, P Y],
$$

for all vector fields $X, Y$ on $M$. As in the case with complex structure, para-complex one $P$ is integrable if and only if $N_{P}=0$. 
Para-Kählerian manifold can be defined as pseudoRiemannian manifold $(M, g)$ with skew-symmetric paracomplex structure $P$, that is parallel with respect to the Levi-Civita connection. If $(g, P)$ is a para-Kählerian structure on $M$, then $\omega=g \circ P$ is symplectic structure, and eigen-distributions $T^{ \pm} M$, corresponding to eigen-values \pm 1 of field $P$, represent two integrable $\omega$-Lagrangian distributions. Therefore the para-Kählerian structure can be identified with bi-Lagrangian structure $\left(\omega, T^{ \pm} M\right)$, where $\omega$ is a symplectic structure, and $T^{ \pm} M$ are the integrable Lagrangian distributions. In [1] presents a review of the theory para-complex structures, and the invariant para-complex and para-Kählerian structures on homogeneous spaces of semi-simple Lie groups are considered in detail. It is shown that every invariant para-Kähler structure $P$ on $M=G / H$ defines a unique para-Kähler Einstein structure $(g, P)$ with given non-zero scalar curvature.

Since the 2-form $\omega$ is not closed, it is possible to consider the 3-form $d \omega$. In [6] Hitchin had defined the concept of non-degeneracy (stability) for 3-forms $\Omega$ and built a linear operator $K_{\Omega}$, whose square is proportional to identity operator $I d$. Recall the basic Hitchin's constructions.

Let $V$ be a six-dimensional real vector space, $\mu$ be a form of volume on $V$, and $\Lambda^{3} V^{*}$ be a 20-dimensional linear space of skew-symmetric 3-forms on $V$. We shall take interior product $l_{X} \Omega \in \Lambda^{2} V^{*}$ for the form $\Omega \in \Lambda^{3} V^{*}$ and vector $X \in V$. Then $l_{X} \Omega \wedge \Omega \in \Lambda^{5} V^{*}$. Natural pairing by the exterior product $V^{*} \otimes \Lambda^{5} V^{*} \rightarrow \Lambda^{6} V^{*} \cong \mathbf{R} \mu$ defines the isomorphism $A: \Lambda^{5} V^{*} \rightarrow V$. Using $\Lambda^{5} V^{*} \cong V$ we define linear map $K_{\Omega}: V \rightarrow V$ as

$$
K_{\Omega}(X)=A\left(l_{X} \Omega \wedge \Omega\right) .
$$

In other words, $\imath_{K \Omega(X)} \mu=l_{X} \Omega \wedge \Omega$. Define $\lambda(\Omega) \in \mathbf{R}$ as a trace of the square of $K_{\Omega}$ :

$$
\lambda(\Omega)=\frac{1}{6} \operatorname{tr} K_{\Omega}^{2},
$$

The form $\Omega$ is called non-degenerated (or stable) if $\lambda(\Omega) \neq 0$.

It is shown in [6] that if $\lambda(\Omega) \neq 0$, then

- $\lambda(\Omega)>0$ if and only if $\Omega=\alpha+\beta$, where $\alpha, \beta$ are real decomposable 3-forms and $\alpha \wedge \beta \neq 0$;

- $\lambda(\Omega)<0$ if and only if $\Omega=\alpha+\bar{\alpha}$, where $\alpha \in$ $\Lambda^{3}\left(V^{*} \otimes \mathbf{C}\right)$ is complex decomposable 3-form and $\alpha \wedge \bar{\alpha} \neq 0$.

It follows that if $\Omega$ is real and $\lambda(\Omega)>0$, then it lies in $G L(V)$-orbit of form $\varphi=\theta^{1} \wedge \theta^{2} \wedge \theta^{3}+\theta^{4} \wedge \theta^{5} \wedge \theta^{6}$ for some basis $\theta^{1}, \ldots, \theta^{6}$ in $V^{*}$, and if $\lambda(\Omega)<0$, then it lies in orbit of form $\varphi=\alpha+\bar{\alpha}$, where $\alpha=$ $\left(\theta^{1}+i \theta^{2}\right) \wedge\left(\theta^{3}+i \theta^{4}\right) \wedge\left(\theta^{5}+i \theta^{6}\right)$.

Then the real 20-dimensional vector space $\Lambda^{3}\left(V^{*}\right)$ contains invariant quadratic hypersurface $\lambda(\Omega)=0$ dividing the $\Lambda^{3}\left(V^{*}\right)$ to 2 open sets: $\lambda(\Omega)>0$ and $\lambda(\Omega)<0$. The component of the unit of the stabilizer of the 3 -form lying in the first set is conjugate to the group $S L(3, \mathbf{R}) \times S L(3, \mathbf{R})$, and in the other case to the group $S L(3, \mathbf{C})$. The linear transformation of $K_{\Omega}$ has [6] the following properties: $\operatorname{tr} K_{\Omega}=0$ and $K_{\Omega}^{2}=\lambda(\Omega) I d$. In the case $\lambda(\Omega)<0$, the real 3-form $\Omega$ defines the structure $J_{\Omega}$ 90 of complex vector space on real vector space $V$ as follows:

$$
J_{\Omega}=\frac{1}{\sqrt{-\lambda(\Omega)}} K_{\Omega}
$$

But if $\lambda(\Omega)>0$, 3-form $\Omega$ defines the para-complex structure $J_{\Omega}$, i.e. $J_{\Omega}{ }^{2}=1, J_{\Omega} \neq 1$ on real vector space $V$ by similar formula:

$$
J_{\Omega}=\frac{1}{\sqrt{\lambda(\Omega)}} K_{\Omega} \text {. }
$$

Recall that the structure of almost a product is called para-complex, if eigen-subspaces have the same dimension.

The elements of $G L(V)$ orbits of 3-form $\Omega$, corresponding to $\lambda(\Omega)>0$, have stabilizer $S L(3, \mathbf{R}) \times S L(3, \mathbf{R})$ in $G L^{+}(V)$ and $J_{\Omega}$ is para-complex structure, i.e. $J_{\Omega}{ }^{2}=1, J_{\Omega} \neq 1$. The elements of orbit corresponding to $\lambda(\Omega)<0$, have stabilizer $S L(3, \mathbf{C})$ in $G L^{+}(V)$ and $J_{\Omega}$ is almost complex structure, i.e. $J_{\Omega}{ }^{2}=-1$. In both cases, dual to $\Omega$ form is defined by formula $\Omega^{\wedge}=J_{\Omega^{*}}^{*}$. If $\lambda(\Omega)>0$ and $\Omega=\alpha+\beta$, then $\Omega^{\wedge}=\alpha-\beta$. But if $\lambda(\Omega)<0$ and $\Omega=\alpha+\bar{\alpha}$, then $\Omega^{\wedge}=i(\bar{\alpha}-\alpha)$. Note that $\Omega^{\wedge}=-\Omega$ in both cases and $J_{\Omega^{\wedge}}=-\varepsilon J_{\Omega}$, where $\varepsilon$ is the sign of $\lambda(\Omega)$. The additional 3 -form $\Omega \wedge$ has a defining property: if $\lambda(\Omega)>0$, then $\Omega+\Omega \wedge$ is decomposable, and if $\lambda(\Omega)<0$, then complex form $\Psi=\Omega$ $+i \Omega$ is decomposable.

The pair $(\omega, \Omega) \in \Lambda^{2}\left(V^{*}\right) \times \Lambda^{3}\left(V^{*}\right)$ non-degenerated forms is called compatible if $\omega \wedge \Omega=0$ (or, equivalently, $\Omega^{\wedge} \wedge \omega=0$ ), and it is called normalized, if $\hat{\Omega} \wedge \Omega=2 \omega^{3} / 3$.

Each compatible pair $(\omega, \Omega)$ uniquely defines $\varepsilon$ complex structure $J_{\Omega}$ (i.e. $J_{\Omega}{ }^{2}=\varepsilon$ ), scalar product $g_{(\omega, \Omega)}(X, Y)=\omega\left(X, J_{\Omega} Y\right)$ (signatures $(3,3)$ for $\varepsilon=1$ and signatures $(2,4)$ or $(4,2)$ for $\varepsilon=-1)$, and (para-)complex volume form $\Psi=\Omega+i_{\varepsilon} \Omega^{\wedge}$ of type $(3,0)$ with respect to $J_{\Omega}$ (where $i_{\varepsilon}$ is a complex or para-complex imaginary unit). In addition, the stabilizer of $(\omega, \Omega)$ pair is $S U(p, q)$ for $\varepsilon=-1$ and $S L(3, \mathbf{R}) \subset S O(3,3)$ for $\varepsilon=1$. Therefore, $(\omega, \Omega)$ pair for $\varepsilon=-1$ defines pseudo almost Hermitian structure. But if $\varepsilon=1$, it defines almost para-Hermitian structure. Such structures are also called special almost $\varepsilon$-Hermitian.

Also recall that $S U(3)$ structure on real sixdimensional almost Hermitian manifold $(M, g, J, \omega)$ is specified by $(3,0)$ form $\Psi$. Almost Hermitian 6-manifold is called half-flat [3] if it admits a reduction to $S U(3)$, for which $d \operatorname{Re} \Psi=0$ and $\omega \wedge d \omega=0$.

In the case of pseudo-Riemannian manifold, each compatible pair $(\omega, \Omega)$ uniquely defines the reduction to $S U(1,2)$ for $\varepsilon=-1$ and $S L(3, \mathbf{R}) \subset S O(3,3)$ for $\varepsilon=1$. Therefore, 6-manifold with a $(\omega, \Omega)$ pair possessing the properties $d \Omega=0$ and $\omega \wedge d \omega=0$, will be called half-flat pseudo almost Hermitian if it admits the reduction to $S U(1,2)$ or half-flat almost para-complex if it admits the reduction to $S L(3, \mathbf{R}) \subset S O(3,3)$.

Remark. In this work, we assume that exterior product and exterior differential are defined without normalizing constant. In particular, then $d x \wedge d y=d x \otimes d y-$ $d y \otimes d x$ and $d \eta(X, Y)=X \eta(Y)-Y \eta(X)-\eta([X, Y])$. Let $\nabla$ be 
the Levi-Civita connectivity corresponding to leftinvariant (pseudo)Riemannian metric $g$. It is defined by six-membered formula [7], which becomes the following form for left-invariant vector fields $X, Y, Z$ on Lie group: $2 g\left(\nabla_{X} Y, Z\right)=g([X, Y], Z)+g([Z, X], Y)+g(X,[Z, Y])$. If $R(X, Y)=\left[\nabla_{X}, \nabla_{Y}\right]-\nabla_{[X, Y]}$ is a curvature tensor, then Ricci tensor $\operatorname{Ric}(X, Y)$ for (pseudo)Riemannian metric $g$ is defined as a construction of a curvature tensor over the first and fourth (upper) indices.

\section{RESULTS AND DISCUSSION}

In this section Lie groups that do not admit neither symplectic, nor left-invariant complex structures will be considered. Such Lie groups will be called singular. It will be shown that they admit non-degenerated leftinvariant 2-forms, whose exterior differentials are nondegenerated. In addition, they admit almost paracomplex structures and Einstein pseudo-Riemannian metrics of signature $(3,3)$.

\section{Lie group $G_{1}$}

Singular group $G_{1}$ that does not admit neither symplectic, nor complex structures. Non-zero commutation relations:

$$
\begin{aligned}
{\left[e_{1}, e_{2}\right] } & =e_{3},\left[e_{1}, e_{3}\right]=e_{4},\left[e_{1}, e_{4}\right]=e_{5},\left[e_{2}, e_{3}\right]= \\
& =e_{5},\left[e_{3}, e_{4}\right]=e_{6},\left[e_{2}, e_{5}\right]=-e_{6} .
\end{aligned}
$$

Lie algebra $\mathbf{g}$ has ideals: $C^{1} \mathbf{g}=D^{1} \mathbf{g}=\mathbf{R}\left\{e_{3}, e_{4}, e_{5}, e_{6}\right\}$, $C^{2} \mathbf{g}=\mathbf{R}\left\{e_{4}, e_{5}, e_{6}\right\}, C^{3} \mathbf{g}=\mathbf{R}\left\{e_{5}, e_{6}\right\}, C^{4} \mathbf{g}=Z=\mathbf{R}\left\{e_{6}\right\}$ is the center of Lie algebra. Filiform Lie algebra. Does not admit half-flat structures [3].

Let $\omega=a_{i j} e^{i} \wedge e^{j}$ be any left-invariant 2-form. For the general form $\omega$ Hitchin's operator $K_{\omega}$ has quite complicated form and the following function $\lambda(d \omega)$ :

$$
\begin{gathered}
\lambda=4\left(a_{16} a_{56}{ }^{2}+4 a_{35} a_{56}{ }^{2}+4 a_{36}{ }^{2} a_{56}-4 a_{36} a_{46}{ }^{2}-\right. \\
\left.-4 a_{45} a_{46} a_{56}\right) a_{56}+a_{46}{ }^{4} .
\end{gathered}
$$

Thus, in general, 3-form $d \omega$ is non-degenerated. It is easy to see that 2-form $\omega$ is closed if and only if $a_{16}=a_{26}$ $=a_{36}=a_{35}=a_{45}=a_{46}=a_{56}=0$ and $a_{34}=-a_{25} . a_{24}=a_{15}$. However, such 2-form $\omega$ is degenerated. There are several natural ways to weaken the closedness requirement of the 2 -form $\omega$, so as not to lose the nondegeneracy of $\omega$ and $d \omega$.

Option 1. In that case we will not suppose that coefficients $a_{46}$ and $a_{56}$, which essentially occur in the expression for function $\lambda(d \omega)$, are non-zero. Moreover, we will suppose that $a_{56} \neq 0$. Then the property $\omega \wedge d \omega=0$ is fulfilled under condition $a_{15}=0, a_{25}=0$ and $a_{12} a_{56}=a_{13} a_{46}, a_{23}=-a_{14}$. 2-form $\omega$ is non-degenerated under condition that $a_{14} a_{56} \neq 0$ and $\omega$ and $d \omega$ become

$$
\begin{gathered}
\omega=e^{1} \wedge\left(a_{13} a_{46} / a_{56} e^{2}+a_{13} e^{3}+a_{14} e^{4}\right)- \\
-a_{14} e^{2} \wedge e^{3}+a_{46} e^{4} \wedge e^{6}+a_{56} e^{5} \wedge e^{6}, \\
d \omega=-a_{46} e^{136}+a_{46} e^{245}-a_{56} e^{146}-a_{56} e^{236}+a_{56} e^{345} .
\end{gathered}
$$

The function $\lambda(d \omega)$ of the Hitchin's operator [6] for 3-form $d \omega$ becomes $\lambda=a_{46}{ }^{4}$. The Hitchin's operator $K_{d \omega}$ has a matrix

$$
K_{d \omega}=\left[\begin{array}{cccccc}
-a_{46}^{2} & -2 a_{46} a_{56} & -2 a_{56}^{2} & 0 & 0 & 0 \\
0 & a_{46}^{2} & 2 a_{46} a_{56} & 2 a_{56}^{2} & 0 & 0 \\
0 & 0 & -a_{46}^{2} & -2 a_{46} a_{56} & 0 & 0 \\
0 & 0 & 0 & a_{46}^{2} & 0 & 0 \\
0 & 0 & 0 & 0 & a_{46}^{2} & 2 a_{56}^{2} \\
0 & 0 & 0 & 0 & 0 & -a_{46}^{2}
\end{array}\right]
$$

Determine the operator $P=K_{d \omega} / a_{46}^{2}$. It defines leftinvariant almost para-complex structure $P^{2}=I d$, having the property $\omega(P X, P Y)=-\omega(X, Y)$. Eigensubspaces $E^{ \pm}$related to the eigen-values \pm 1 of operator $P$ are generated by the following vectors:

$$
\begin{gathered}
E^{+}=\left\{a_{56}{ }^{3} e^{1}-a_{56} a_{46}{ }^{2} e^{3}+a_{46}{ }^{3} e^{4},-a_{56} e^{1}+a_{46} e^{2}, e^{5}\right\}, \\
E^{-}=\left\{e^{1},-a_{56} e^{2}+a_{46} e^{3},-a_{56}{ }^{2} e^{5}+a_{46}{ }^{2} e^{6}\right\} .
\end{gathered}
$$

It is easy to see that they are not closed relative to Lie bracket, so $P$ defines non-integrable almost a paracomplex structure.

Define pseudo-Riemannian metric $g(X, Y)=\omega(X, P Y)$ of signature $(3,3)$. It is given by:

$$
\begin{gathered}
g=2 e^{1} \cdot\left(a_{13} a_{46} / a_{56} e^{2}+a_{13} e^{3}+a_{14} e^{4}\right)+ \\
+2 e^{2} \cdot\left(a_{13} e^{2}+\left(2 a_{13} a_{56}+a_{14} a_{46}\right) / a_{46} e^{3}+2 a_{14} a_{56} / a_{46} e^{4}\right)+ \\
\left.+2 e^{3} \cdot\left(a_{56}\left(a_{13} a_{56}+a_{14} a_{46}\right) / a_{46}{ }^{2} e^{3}+2 a_{14} a_{56}{ }^{2}\right) / a_{46}{ }^{2} e^{4}\right)- \\
-2 a_{46} e^{4} \cdot e^{6}-2 a_{56} e^{5} \cdot e^{6}-2 a_{56}{ }^{3} / a_{46}{ }^{2} e^{6} \cdot e^{6} .
\end{gathered}
$$

Direct calculations of curvature tensor in Maple system show that this metric is not Einsteinian and that it has scalar curvature

$$
R=\frac{3\left(8 a_{13} a_{56}^{7}-8 a_{14} a_{46} a_{56}^{6}-a_{46}^{8}\right)}{a_{56} a_{14}^{2} a_{46}^{6}} .
$$

Option 2. Take the 2-form $\omega$ in the view $\omega=\omega_{0}+\omega_{C}$, where $\omega_{0}$ is a closed 2-form and $\omega_{C}$ is a non-degenerated 2-form on the ideality $C^{2} \mathrm{~g}=\mathbf{R}\left\{e_{4}, e_{5}, e_{6}\right\}$. We require that the 2-form $\omega$ should have the property $\omega \wedge d \omega=0$ :

$$
a_{25}=0, a_{15}=0, a_{12} a_{56}=a_{13} a_{46}, a_{56} a_{23}+a_{14} a_{56}=0 .
$$

Then $\omega$ is non-degenerated under condition $a_{14} a_{56} \neq 0$. The $\omega$ and $d \omega$ take the view:

$$
\begin{gathered}
\omega=e^{1} \wedge\left(a_{13} a_{46} / a_{56} e^{2}+a_{13} e^{3}+a_{14} e^{4}\right)-a_{14} e^{2} \wedge e^{3}+a_{45} \\
e^{4} \wedge e^{5}+a_{46} e^{4} \wedge e^{6}+a_{56} e^{5} \wedge e^{6}, \\
d \omega=a_{45} e^{234}-a_{45} e^{135}-a_{46} e^{136}+a_{46} e^{245}-a_{56} e^{146}- \\
a_{56} e^{236}+a_{56} e^{345} .
\end{gathered}
$$

In this case, $\lambda(d \omega)$ function is expressed by the $\lambda=$ $a_{46}{ }^{4}-4 a_{46} a_{45} a_{56}{ }^{2}$ and it can take both positive and negative values.

Case 1. The function $\lambda(d \omega)$ takes the value -1 when $a_{45}=\left(a_{46}{ }^{4}+1\right) /\left(4 a_{46} a_{56}{ }^{2}\right)$. Then the operator $J=K_{d \omega}$ defines almost complex structure compatible with $\omega$ and has the form: 


$$
J=\left[\begin{array}{cccccc}
-a_{46}^{2} & -2 a_{46} a_{56} & -2 a_{56}^{2} & 0 & 0 & 0 \\
\frac{a_{46}^{4}+1}{2 a_{46} a_{56}} & a_{46}^{2} & 2 a_{46} a_{56} & 2 a_{56}^{2} & 0 & 0 \\
0 & 0 & -a_{46}^{2} & -2 a_{46} a_{56} & 0 & 0 \\
0 & 0 & \frac{a_{46}^{4}+1}{2 a_{46} a_{56}} & a_{46}^{2} & 0 & 0 \\
0 & 0 & -\frac{a_{46}^{4}+1}{2 a_{56}^{2}} & -\frac{a_{46}^{4}+1}{2 a_{46} a_{56}} & a_{46}^{2} & 2 a_{56}^{2} \\
0 & 0 & \frac{\left(a_{46}^{4}+1\right)^{2}}{8 a_{46}^{2} a_{56}^{4}} & 0 & -\frac{a_{46}^{4}+1}{2 a_{56}^{2}} & -a_{46}^{2}
\end{array}\right]
$$

Specify the associated pseudo-Riemannian metric by formula $g(X, Y)=\omega(X, J Y)$ of signature $(2,4)$. Direct calculations of curvature tensor in Maple system show that this metric is not Einsteinian and that it has scalar curvature

$$
R=\frac{8 a_{13} a_{56}^{7}-8 a_{14} a_{46} a_{56}^{6}-1}{a_{56} a_{14}^{2}}
$$

Case 2. The function $\lambda(d \omega)$ takes the value +1 when $a_{45}=\left(a_{46}{ }^{4}-1\right) /\left(4 a_{46} a_{56}{ }^{2}\right)$. Then the operator $P=K_{d \omega}$ defines almost para-complex structure compatible with $\omega$ and $P$ has the same matrix, as the above almost complex structure $J$ has, where it is necessary to substitute $a_{46}^{4}-1$ instead of $a_{46}^{4}+1$. The corresponding metric $g(X, Y)=\omega(X, P Y)$ is pseudo-Riemannian of signature $(3,3)$; it is not the Einsteinian one and has the same scalar curvature, as in the first case.

Conclusions. Any left-invariant closed 2-form $\omega$ on Lie group $G_{1}$ is degenerated. There are several ways to weaken the closedness requirement of $\omega$ to preserve non-degeneracy $\omega$, in ways that 3 -form $d \omega$ is nondegenerated and the property $\omega \wedge d \omega=0$ is fulfilled. Hitchin's operator $K_{d \omega}$ corresponding to nondegenerated 3-form $d \omega$, can define either almost complex structure, or almost para-complex, depending on the chosen $\omega$. Associated metric $g(X, Y)=\omega\left(X, J_{d \omega} Y\right)$ is pseudo-Riemannian of signature $(2,4)$ or $(3,3)$. As a result, we have obtained a compatible pair $(\omega, \Omega)$, where $\Omega=d \omega$. Therefore, the properties $d \Omega=0$ and $\omega \wedge d \omega=0$ are fulfilled in an obvious way. The $(3,0)$-form has a view of $\Psi=d \omega+i_{\varepsilon} d \omega{ }^{\wedge}$, where $i_{\varepsilon}$ is a complex or para-complex unit. Thus, half-flat pseudo almost Hermitian and half-flat para-complex structures were naturally defined on Lie group $G_{1}$.

\section{Lie group $\boldsymbol{G}_{2}$}

Singular group $G_{2}$ that does not admit neither symplectic, nor complex structures. Commutation relations

$$
\begin{gathered}
{\left[e_{1}, e_{2}\right]=e_{3},\left[e_{1}, e_{3}\right]=e_{4},\left[e_{1}, e_{4}\right]=e_{5},\left[e_{3}, e_{4}\right]=e_{6},} \\
{\left[e_{2}, e_{5}\right]=-e_{6} .}
\end{gathered}
$$

Lie algebra $\mathbf{g}$ has ideals: $C^{1} \mathbf{g}=D^{1} \mathbf{g}=\mathbf{R}\left\{e_{3}, e_{4}, e_{5}, e_{6}\right\}$, $C^{2} \mathbf{g}=\mathbf{R}\left\{e_{4}, e_{5}, e_{6}\right\}, C^{3} \mathbf{g}=\mathbf{R}\left\{e_{5}, e_{6}\right\}, C^{4} \mathbf{g}=Z=\mathbf{R}\left\{e_{6}\right\}$ is the center of Lie algebra. Filiform Lie algebra. Does not admit half-flat structures [3].

Let $\omega=a_{i j} e^{i} \wedge e^{j}$ be any left-invariant non-degenerated 2-form. For such a generic 2-form the square of the Hitchin's operator [6] for 3-form $d \omega$ has a diagonality:
$K_{d \omega}=\left(a_{46}{ }^{2}-2 a_{36} a_{56}\right)^{2} I d$. Therefore, 3-form $d \omega$ is nondegenerated if $\lambda(d \omega)=a_{46}{ }^{2}-2 a_{36} a_{56} \neq 0$. The 2-form $\omega$ is closed only in the case when it has the form:

$$
\begin{gathered}
\omega=e^{1} \wedge\left(a_{12} e^{2}+a_{13} e^{3}+a_{14} e^{4}+a_{15} e^{5}\right)+e^{2} \wedge\left(a_{23} e^{3}-\right. \\
\left.-a_{34} e^{5}\right)+a_{34} e^{3} \wedge e^{4} .
\end{gathered}
$$

Such 2-form $\omega$ is non-degenerated. In order to preserve the non-degeneracy of the $\omega$ and $d \omega$ at the minimal weakening of closedness property of $\omega$, two variants are possible: $a_{46} \neq 0$, or $a_{36} \neq 0$ and $a_{56} \neq 0$. However, if $a_{56} \neq 0$, then simple calculations show that the property $\omega \wedge d \omega=0$ is incompatible with the nondegeneracy $\omega$.

Therefore, consider a case when $a_{46} \neq 0$. Then $K_{d \omega}=a_{46}{ }^{4} I d$. In addition, $\omega \wedge d \omega=0$ under condition that $a_{13}=0$ and $a_{34}=0$. Then the 2-form $\omega$ is nondegenerated under condition $a_{23} a_{15} a_{46} \neq 0$, and the $\omega$ and $d \omega$ take the view:

$$
\begin{gathered}
\omega=e^{1} \wedge\left(a_{12} e^{2}+a_{14} e^{4}+a_{15} e^{5}\right)+a_{23} e^{2} \wedge e^{3}+a_{46} e^{4} \wedge e^{6}, \\
d \omega=a_{46}\left(-e^{136}+e^{245}\right) .
\end{gathered}
$$

The operator $K_{d \omega}$ for the 3-form $d \omega$ has the diagonal form: $K_{d \omega}=\operatorname{diag}\left\{-a_{46}{ }^{2}, a_{46}{ }^{2},-a_{46}{ }^{2}, a_{46}{ }^{2}, a_{46}{ }^{2},-a_{46}{ }^{2}\right\}$. Define the operator $P=K_{d \omega} / a_{46}^{2}$. It defines leftinvariant almost para-complex structure $P^{2}=I d$, having the property $\omega(P X, P Y)=-\omega(X, Y)$. Eigen-subspaces $E^{ \pm}$related to the eigen-values \pm 1 of operator $P$ are generated by the following vectors:

$$
E^{+}=\left\{e^{2}, e^{4}, e^{5}\right\}, E^{-}=\left\{e^{1}, e^{3}, e^{6}\right\} .
$$

It is easy to see that they are not closed relative to Lie bracket, so $P$ defines non-integrable almost a paracomplex structure.

Define the pseudo-Riemannian metric $g(X, Y)=\omega(X$, $P Y)$. It has a signature $(3,3)$ and it is given by:

$$
g=2 e^{1} \cdot\left(a_{12} e^{2}+a_{14} e^{4}+a_{15} e^{5}\right)-2 a_{23} e^{2} \cdot e^{3}-2 a_{46} e^{4} \cdot e^{6} .
$$

Direct calculations in a Maple system show that this metric is Einsteinian and that its Ricci tensor and scalar curvature are specified by formulas:

$$
\text { Ric }=\frac{a_{46}}{2 a_{15} a_{23}} g \quad R=-\frac{3 a_{46}}{a_{15} a_{23}} .
$$

\section{Lie group $G_{3}$}

Singular group $G_{3}$ that does not admit neither symplectic, nor complex structures. Commutation relations

$$
\left[e_{1}, e_{2}\right]=e_{4},\left[e_{1}, e_{3}\right]=e_{5},\left[e_{1}, e_{4}\right]=e_{6},\left[e_{3}, e_{5}\right]=e_{6} .
$$

Lie algebra $\mathbf{g}$ has ideals: $C^{1} \mathbf{g}=D \mathbf{g}=\mathbf{R}\left\{e_{4}, e_{5}, e_{6}\right\}$, $C^{2} \mathbf{g}=\mathbf{R}\left\{e_{6}\right\}=Z$ is the center of Lie algebra Does admit half-flat structure [3].

Let $\omega=a_{i j} e^{i} \wedge e^{j}$ be any 2-form. The Hitchin's operator $K_{d \omega}$ for generic 2-form $\omega$ has a quite complicated view. Moreover, $K_{d \omega}{ }^{2}=a_{46}^{4} I d$. For $\lambda=a_{46}^{4} \neq 0$ the 3 -form $d \omega$ is non-degenerated. The operator $P=K_{d \omega} / a_{46}{ }^{2}$ defines the left-invariant almost para-complex structure on $\mathbf{g}$. The 
$\omega \wedge d \omega=0$ property is fulfilled under the following conditions:

$$
\begin{gathered}
a_{12} a_{46}-a_{14} a_{26}-a_{23} a_{56}+a_{24} a_{16}+a_{25} a_{36}-a_{35} a_{26}=0, \\
a_{25} a_{46}-a_{24} a_{56}-a_{26} a_{45}=0, a_{35} a_{46}-a_{36} a_{45}- \\
-a_{34} a_{56}=0 .
\end{gathered}
$$

It is easy to see that the 2 -form $\omega$ is closed only if

$$
\begin{gathered}
\omega=e^{1} \wedge\left(a_{12} e^{2}+a_{13} e^{3}+a_{14} e^{4}+a_{15} e^{5}\right)+ \\
+e^{2} \wedge\left(a_{23} e^{3}+a_{24} e^{4}+a_{25} e^{5}\right)+e^{3} \wedge\left(a_{25} e^{4}+a_{35} e^{5}\right) .
\end{gathered}
$$

In order to preserve the non-degeneracy of the $\omega$ and $d \omega$ at the minimal weakening of closedness property of $\omega$, consider the case when $a_{46} \neq 0$. The $\omega \wedge d \omega=0$ property is fulfilled under the condition $a_{12}=0, a_{25}=0, a_{35}=0$. Thus, 2 -form $\omega$ is non-degenerated if $a_{15} a_{23} a_{46} \neq 0$ and then we obtain:

$$
\begin{gathered}
\omega=e^{1} \wedge\left(a_{13} e^{3}+a_{14} e^{4}+a_{15} e^{5}\right)+e^{2} \wedge\left(a_{23} e^{3}+a_{24} e^{4}\right)+ \\
+a_{46} e^{4} \wedge e^{6}, d \omega=-a_{46}\left(e^{126}+e^{345}\right) .
\end{gathered}
$$

The operator $K_{d \omega}$ for the 3-form $d \omega$ has the diagonal view, $K_{d \omega}=\operatorname{diag}\left\{-a_{46}{ }^{2},-a_{46}{ }^{2}, a_{46}{ }^{2}, a_{46}{ }^{2}, a_{46}{ }^{2},-a_{46}{ }^{2}\right\}$. Define the operator $P=K_{d \omega} / a_{46}^{2}$. It defines leftinvariant almost para-complex structure $P^{2}=I d$, having the property $\omega(P X, P Y)=-\omega(X, Y)$. Eigen-subspaces $E^{ \pm}$related to the eigen-values \pm 1 of operator $P$ are generated by the following vectors:

$$
E^{+}=\left\{e^{3}, e^{4}, e^{5}\right\}, E^{-}=\left\{e^{1}, e^{2}, e^{6}\right\} .
$$

It is easy to see that they are not closed relative to Lie bracket, so $P$ sets non-integrable almost a paracomplex structure.

Define the pseudo-Riemannian metric $g(X, Y)=\omega(X$, $P Y)$ of signature $(3,3)$. It is given by:

$$
\begin{gathered}
g=2 e^{1} \cdot\left(a_{13} e^{3}+a_{14} e^{4}+a_{15} e^{5}\right)+2 a_{23} e^{2} \cdot e^{3}+2 a_{24} e^{2} \cdot e^{4}- \\
-2 a_{46} e^{4} \cdot e^{6} .
\end{gathered}
$$

Direct calculations in a Maple system show that this metric is Einsteinian and that its Ricci tensor and scalar curvature are specified by formulas:

$$
\text { Ric }=\frac{a_{46}}{2 a_{15} a_{23}} g \quad R=-\frac{3 a_{46}}{a_{15} a_{23}} .
$$

\section{Lie group $G_{4}$}

Singular group that does not admit neither symplectic, nor complex structures. Commutation relations:

$$
\left[e_{1}, e_{2}\right]=e_{4},\left[e_{2}, e_{3}\right]=e_{5},\left[e_{1}, e_{4}\right]=e_{6},\left[e_{3}, e_{5}\right]=e_{6} \text {. }
$$

Lie algebra $\mathbf{g}$ has ideals: $C^{1} \mathbf{g}=D^{1} \mathbf{g}=\mathbf{R}\left\{e_{4}, e_{5}, e_{6}\right\}$, $C^{2} \mathbf{g}=\mathbf{R}\left\{e_{6}\right\}=Z$ is the center of Lie algebra. Does admit half-flat structure [3].

Let $\omega=a_{i j} e^{i} \wedge e^{j}$ be any 2-form. The Hitchin's operator $K_{d \omega}$ for generic 2-form $\omega$ has a quite complicated form. Moreover, $K_{d \omega}{ }^{2}=\left(a_{46}{ }^{2}-a_{56}{ }^{2}\right)^{2} I d$. The $\omega \wedge d \omega=0$ property is fulfilled under the following conditions:

$-a_{12} a_{46}+a_{14} a_{26}-a_{16} a_{24}+a_{23} a_{56}-a_{25} a_{36}+a_{26} a_{35}=0$,

$$
\begin{gathered}
-a_{14} a_{56}+a_{15} a_{46}-a_{16} a_{45}=0, \\
a_{34} a_{56}-a_{35} a_{46}+a_{36} a_{45}=0 .
\end{gathered}
$$

It is easy to see that the 2-form $\omega$ is closed only if

$$
\begin{gathered}
\omega=e^{1} \wedge\left(a_{12} e^{2}+a_{13} e^{3}+a_{14} e^{4}+a_{15} e^{5}\right)+ \\
+e^{2} \wedge\left(a_{23} e^{3}+a_{24} e^{4}+a_{25} e^{5}\right)+e^{3} \wedge\left(-a_{15} e^{4}+a_{35} e^{5}\right) .
\end{gathered}
$$

In order to preserve the non-degeneracy of the $\omega$ and $d \omega$ at the minimal weakening of closedness property of $\omega$, consider the case when $a_{46} \neq 0$ and $a_{56} \neq 0$. The $\omega \wedge d \omega=0$ property is fulfilled under the following conditions:

$$
\begin{gathered}
-a_{12} a_{46}+a_{23} a_{56}=0,-a_{14} a_{56}+a_{15} a_{46}=0, \\
-a_{15} a_{56}-a_{35} a_{46}=0
\end{gathered}
$$

and $d \omega$ is given by the sum of two decomposable 3-forms:

$$
d \omega=\left(a_{56} e^{1}-a_{46} e^{3}\right) \wedge e^{45}+\left(-a_{46} e^{1}+a_{56} e^{3}\right) \wedge e^{26} .
$$

The function $\lambda(d \omega)$ of the Hitchin's operator for 3 -form $d \omega$ has the same view $\lambda=\left(a_{46}{ }^{2}-a_{56}{ }^{2}\right)^{2}$. And operator $K_{d \omega}$ is given by:

$$
\begin{gathered}
K_{d \omega}=\left(-a_{46}{ }^{2}-a_{56}{ }^{2}\right) e_{1} \otimes e^{1}+\left(-a_{46}{ }^{2}+a_{56}{ }^{2}\right) e_{2} \otimes e^{2}+\left(a_{46}{ }^{2}+\right. \\
\left.+a_{56}{ }^{2}\right) e_{3} \otimes e^{3}+\left(a_{46}{ }^{2}-a_{56}{ }^{2}\right) e_{4} \otimes e^{4}+\left(a_{46}{ }^{2}-a_{56}{ }^{2}\right) e_{5} \otimes e^{5}+ \\
+\left(-a_{46}{ }^{2}+a_{56}{ }^{2}\right) e_{6} \otimes e^{6}+2 a_{46} a_{56} e_{1} \otimes e^{3}-2 a_{46} a_{56} e_{3} \otimes e^{1} .
\end{gathered}
$$

Define the operator $P=K_{d \omega} /\left|a_{46}^{2}-a_{56}^{2}\right|$. It defines left-invariant almost para-complex structure $P^{2}=I d$, having the property $\omega(P X, P Y)=-\omega(X, Y)$. Eigensubspaces related to eigen-values $\left(a_{46}{ }^{2}-a_{56}{ }^{2}\right) /\left|a_{46}{ }^{2}-a_{56}{ }^{2}\right|$ and $\left(a_{56}{ }^{2}-a_{46}{ }^{2}\right) /\left|a_{46}{ }^{2}-a_{56}{ }^{2}\right|$ of the operator $P$ are generated by the following vectors:

$$
E_{1}=\left\{a_{56} e^{1}+a_{46} e^{3}, e^{4}, e^{5}\right\}, E_{2}=\left\{a_{46} e^{1}+a_{56} e^{3}, e^{2}, e^{6}\right\} .
$$

It is easy to see that they are not closed relative to Lie bracket, so $P$ sets non-integrable almost a paracomplex structure.

Define the pseudo-Riemannian metric $g(X, Y)=$ $\omega(X, P Y)$ of signature $(3,3)$. Direct calculations in a Maple system show that this metric is Einsteinian and that its Ricci tensor and scalar curvature are specified by formulas:

$$
\begin{gathered}
\text { Ric }=\frac{\left|a_{46}^{2}-a_{45}^{2}\right|}{2 a_{13}\left(a_{24} a_{56}-a_{25} a_{46}\right)} g, \\
R=-\frac{3\left|a_{46}^{2}-a_{45}^{2}\right|}{a_{13}\left(a_{24} a_{56}-a_{25} a_{46}\right)} .
\end{gathered}
$$

In particular case, when one of the arguments $a_{46}$ and $a_{56}$ is equal to zero, the situation becomes much simpler. For example, let $a_{56}=0$. The property $\omega \wedge d \omega=0$ is fulfilled under the following conditions: $a_{12}=0, a_{15}=0$, $a_{35}=0$. Then 2-form is non-degenerated under the condition $a_{13} a_{25} a_{46} \neq 0$, and we obtain:

$$
\omega=e^{1} \wedge\left(a_{13} e^{3}+a_{14} e^{4}\right)+e^{2} \wedge\left(a_{23} e^{3}+a_{24} e^{4}+a_{25} e^{5}\right)+a_{46} e^{4} \wedge e^{6},
$$$$
d \omega=-a_{46} e^{126}-a_{46} e^{345},
$$ 


$$
K_{d \omega}=\operatorname{diag}\left\{-a_{46}{ }^{2},-a_{46}{ }^{2}, a_{46}^{2}, a_{46}^{2}, a_{46}^{2},-a_{46}^{2}\right\} .
$$

Define the operator $P=K_{d \omega} / a_{46}^{2}$. It specifies almost para-complex structure $P^{2}=I d$, possessing the property $\omega(P X, P Y)=-\omega(X, Y)$. Eigen-subspaces related to the eigen-values \pm 1 of operator $P$ are generated by the following vectors:

$$
E^{+}=\left\{e^{3}, e^{4}, e^{5}\right\}, E=\left\{e^{1}, e^{2}, e^{6}\right\}
$$

It is easy to see that they are not closed relative to Lie bracket, so $P$ sets non-integrable almost a paracomplex structure.

The pseudo-Riemannian metric $g(X, Y)=\omega(X, P Y)$ of signature $(3,3)$ is given by

$$
\begin{aligned}
g=-2 e^{1} \cdot\left(a_{13} e^{3}+a_{14} e^{4}\right. & \left.+a_{15} e^{5}\right)-2 e^{2} \cdot\left(a_{23} e^{3}+a_{24} e^{4}+a_{25} e^{5}\right)+ \\
& +2 a_{46} e^{4} \cdot e^{6} .
\end{aligned}
$$

Direct calculations in a Maple system show that this metric is Einsteinian and that its Ricci tensor and scalar curvature are specified by formulas:

$$
\text { Ric }=-\frac{a_{46}}{2 a_{13} a_{25}} g \quad R=\frac{3 a_{46}}{a_{13} a_{25}} .
$$

\section{Lie group $\boldsymbol{G}_{5}$}

Singular group that does not admit neither symplectic, nor complex structures. Commutation relations:

$$
\left[e_{1}, e_{2}\right]=e_{5},\left[e_{1}, e_{5}\right]=e_{6},\left[e_{3}, e_{4}\right]=e_{6} .
$$

Lie algebra $\mathbf{g}$ has ideals: $C^{1} \mathbf{g}=D^{1} \mathbf{g}=\mathbf{R}\left\{e_{5}, e_{6}\right\}$, $C^{2} \mathbf{g}=\mathbf{R}\left\{e_{6}\right\}=Z$ is the center of Lie algebra. Does admit half-flat structure [3].

Let $\omega=a_{i j} e^{i} \wedge e^{j}$ be any 2-form. The Hitchin's operator $K_{d \omega}$ for generic 2-form $\omega$ is given by a quite complicated form. Moreover, $K_{d \omega}{ }^{2}=a_{56}{ }^{4} I d$. The $\omega \wedge d \omega=0$ property is fulfilled under the following conditions:

$$
\begin{gathered}
a_{34} a_{56}+a_{35} a_{46}-a_{36} a_{45}=0, \\
a_{12} a_{56}-a_{15} a_{26}+a_{16} a_{25}-a_{23} a_{46}+a_{24} a_{36}-a_{26} a_{34}=0 .
\end{gathered}
$$

It is easy to see that the 2 -form $\omega$ is closed only if

$$
\begin{gathered}
\omega=e^{1} \wedge\left(a_{12} e^{2}+a_{13} e^{3}+a_{14} e^{4}+a_{15} e^{5}\right) \\
+e^{2} \wedge\left(a_{23} e^{3}+a_{24} e^{4}+a_{25} e^{5}\right)+a_{34} e^{3} \wedge e^{4} .
\end{gathered}
$$

Such 2-form $\omega$ is non-degenerated. In order to preserve the non-degeneracy of the $\omega$ and $d \omega$ at the minimal weakening of closedness property of $\omega$, consider the case when $a_{56} \neq 0$. Then the property $\omega \wedge d \omega=0$ is fulfilled under the following conditions: $a_{34}=0$ and $a_{12}=0$. The 2-form $\omega$ is non-degenerated under the condition $a_{56}\left(a_{13} a_{24}-a_{14} a_{23}\right) \neq 0$, and the following formulas occur:

$$
\begin{gathered}
\omega=e^{1} \wedge\left(a_{13} e^{3}+a_{14} e^{4}+a_{15} e^{5}\right)+e^{2} \wedge\left(a_{23} e^{3}+a_{24} e^{4}+a_{25} e^{5}\right)+ \\
+a_{56} e^{5} \wedge e^{6}, d \omega=-a_{56} e^{126}+a_{56} e^{345}, \\
K_{d \omega}=a_{56}{ }^{2} \cdot \operatorname{diag}\{+1,+1,-1,-1,-1,+1\} .
\end{gathered}
$$

Define the operator $P=K_{d \omega} / a_{56}^{2}$. It defines leftinvariant almost para-complex structure $P^{2}=I d$, having the property $\omega(P X, P Y)=-\omega(X, Y)$. Eigen-subspaces related to the eigen-values \pm 1 of operator $P$ are generated by the following vectors:

$$
E^{+}=\left\{e^{1}, e^{2}, e^{6}\right\}, E^{-}=\left\{e^{3}, e^{4}, e^{5}\right\} .
$$

It is easy to see that they are not closed relative to Lie bracket, so $P$ sets non-integrable almost a paracomplex structure.

The pseudo-Riemannian metric $g(X, Y)=\omega(X, P Y)$ of signature $(3,3)$ is given by

$$
\begin{aligned}
g=-2 e^{1} \cdot\left(a_{13} e^{3}+a_{14} e^{4}\right. & \left.+a_{15} e^{5}\right)-2 e^{2} \cdot\left(a_{23} e^{3}+a_{24} e^{4}+a_{25} e^{5}\right)+ \\
& +2 a_{46} e^{4} \cdot e^{6} .
\end{aligned}
$$

Direct calculations in a Maple system show that this metric is Einsteinian and that its Ricci tensor and scalar curvature are specified by formulas:

$$
\begin{gathered}
\text { Ric }=\frac{a_{56}}{2\left(a_{13} a_{24}-a_{14} a_{23}\right)} g, \\
R=-\frac{3 a_{56}}{a_{13} a_{24}-a_{14} a_{23}} .
\end{gathered}
$$

Conclusions. Any left-invariant closed 2-form $\omega$ on Lie groups $G_{2}-G_{5}$ is degenerated. When the closedness requirement of $\omega$ is weakened in order to preserve the nondegeneracy of $\omega$ and $d \omega$ and of the property $\omega \wedge d \omega=0$, the Hitchin's operator $K_{d \omega}$ corresponding to $d \omega$, defines almost para-complex structure $P$. Pseudo-Riemannian metric $g(X, Y)=\omega(X, P Y)$ depending on 5 to 7 arguments is of signature $(3,3)$ and is Einsteinian one. As a result, we have obtained a compatible pair $(\omega, \Omega)$, where $\Omega=d \omega$. Therefore, the properties $d \Omega=0$ and $\omega \wedge d \omega=0$ were fulfilled in an obvious way. The para-complex $(3,0)$-form is given by $\Psi=d \omega+i_{\varepsilon} d \omega$, where $i_{\varepsilon}$ is a para-complex unit.

Thus, multiparametric families of Einsteinian almost para-complex half-flat structures were naturally defined on the Lie groups $G_{2}-G_{5}$ and corresponding nilmanifolds. The structural group is reduced to $S L(3, \mathbf{R}) \subset S O(3,3)$.

\section{REFERENCES}

1. Alekseevskiy D.V., Medori C., and Tomassini A. Odnorodnye para-kelerovy mnogoobraziya Eynshteyna [Homogeneous para-Kähler Einstein manifolds]. Uspekhi Matematicheskikh Nauk [Russian Mathematical Surveys], 2009, vol. 64, no. 1, pp. 3-50. DOI: https://doi.org/10.4213/rm9262.

2. Benson C. and Gordon C.S. Kähler and symplectic structures on nilmanifold. Topology, 1988, vol. 27, pp. 513-518.

3. Conti D. Half-flat nilmanifolds. Mathematische Annalen, 2011, vol. 350(1), pp. 155-168. (arXiv:0903.1175 [math.DG])

4. Cordero L.A., Fernández M., and Ugarte L. Pseudo-Kähler metrics on six-dimensional nilpotent Lie algebras. Journal of Geometry and Physics, 2004, vol. 50, pp. 115-137. DOI: https://doi.org/10.1016/j.geomphys.2003.12.003.

5. Goze M., Khakimdjanov Y., and Medina A. Symplectic or contact structures on Lie groups. Differential Geometry and its Applications, 2004, vol. 21, no. 1, pp. 41-54. 
6. Hitchin N.J. The geometry of three-forms in six dimensions. Differential Geometry and its Applications, 2000, vol. 55, pp. 547-576.

7. Kobayashi S. and Nomizu K. Foundations of Differential Geometry. vol. 1 and 2. New York, London: Interscience Publ., 1963.

8. Salamon S. Complex structures on nilpotent Lie algebras. Journal of Pure and Applied Algebra, 2001, vol. 157, pp. 311-333.

9. Smolentsev N. K. Kanonicheskie psevdokelerovy metriki na shestimernykh nil'potentnykh gruppakh Li [Canonical pseudo-Kählerian metrics on six-dimensional nilpotent Lie groups]. Vestnik KemGU [Bulletin of Kemerovo State University], 2011, no. 3/1 (47), pp. 155-168. (arXiv:1310.5395 [math.DG])

10. Canonical almost pseudo-Kähler structures on six-dimensional nilpotent Lie groups. 2013, arXiv: 1311.4248 [math.DG], 26 p.

Nikolay K. Smolentsev

Dr.Sci.(Phys.-Math.), Professor of the Department of Fundamental Mathematics, Kemerovo State University, Kemerovo, Russian Federation 